This document is the accepted manuscript version of the following article:

\title{
Deep Learning for In Situ and Real-Time Quality Monitoring in Additive Manufacturing Using Acoustic Emission
}

\author{
S.A. Shevchik, G. Masinelli, C. Kenel, C. Leinenbach, and K. Wasmer, Member, IEEE
}

\begin{abstract}
Additive manufacturing is considered as a revolution in manufacturing. However, the high expectations face technical difficulties that prevent further penetration into wider industries. The main reason is the lack of process reproducibility and the absence of a reliable and cost-effective process monitoring. This work is a supplement to existing studies in this field and proposes a unique combination of highly sensitive acoustic sensor and machine learning for process monitoring. The acoustic signals from a real powder-bed fusion additive manufacturing process were collected using a Fiber Bragg grating. The process parameters were intentionally tuned to achieve three levels of quality categories, which were related to the porosity contents inside the workpiece. The quality categories were defined as high, medium and poor quality and their corresponding porosity contents were $0.07,0.30$ and $1.42 \%$, respectively. Wavelet spectrograms of the signals and their encoded label representations, obtained from spectral clustering, were taken as features. A deep convolutional neural network was used to classify the features from each category and the classification accuracy ranges between 78 and $91 \%$. Hence, the proposed method has significant industrial potentials for in situ and real-time quality monitoring of additive manufacturing processes since it requires minimum modifications of commercially available industrial machines.
\end{abstract}

Index Terms - Additive manufacturing, acoustic emission, spectral convolutional neural networks, $M$-band wavelets, fiber optical sensors, fiber Bragg grating, FGB, process monitoring, powder-bed fusion additive manufacturing

Manuscript received October 31, 2018; revised February 2, 2019; accepted April 2, 2019. Paper $\mathrm{N}^{\circ}$ TII-18-2874. (Corresponding author: Kilian Wasmer).

All authors are in the Laboratory for Advanced Materials Processing, Empa - Swiss Federal Laboratories for Materials Science and Technology, Thun, Switzerland (e-mails: sergey.shevchik@empa.ch, giulio.masinelli@empa.ch,_christoph.kenel@alumni.ethz.ch, christian.leinenbach@empa.ch, kilian.wasmer@empa.ch).

Copyright (c) 2009 IEEE. Personal use of this material is permitted. However, permission to use this material for any other purposes must be obtained from the IEEE by sending a request to pubs-permissions@ieee.org.

Color versions of one or more of the figures in this paper are available online at http://ieeexplore.ieee.org.

Digital Object Identifier:

\section{INTRODUCTION}

A dditive manufacturing (AM) is a recent technology for producing 3D items from powder material [1][2]. Although, today, this technology is mainly used for fast prototyping [1], its potential is acknowledged as the next industrial revolution [3] due to several advantages. Those are in a tremendous reduction of geometrical design constraints [4], minimization of the lead-time due to computer-aided design (CAD) [2], the possibility to use different powder materials, or even to design new ones for specific applications. Many industrial sectors are impacted by this new technology. They include industrial machinery, consumer products, electronics, motors and vehicles, medical applications, aerospace, robotics, and others [1][2]. However, the high expectations are tempered due to the technology's immaturity, namely limited repeatability of the workpieces mechanical properties in mass production [1][2]. This raises the demand for in situ and real-time quality monitoring that is not available today [5][6]. The challenge in developing such systems is due to the complexity of the underlying physics that involves a wide range of time scaled events. They embrace not only material heating, melting and solidification, but also phase transformations and residual stresses during the cooling and solidification of the workpiece [1][7]. Those make the workpiece quality sensitive to a very large number of process parameters. They consist of, but not limited to, laser parameters, laser optics, mechanical, thermal and optical material properties, particles configuration in the process zone [1][7]. A slight change of one parameter may significantly impact the quality and/or mechanical properties, characterized by the porosity, cracks and accumulated residual stresses [5][6]. The physical problem is an intricate dependence between the process parameters and the workpiece quality. At present, no physical explanation exists that link all aspects within a single model [5][6]. Thus, most of the AM processes rely more on "trial and error" approach, sorting out and fixing the optimal process parameters within a given range [1][5][6].

To guarantee the workpieces quality and/or mechanical properties, a control is often carried out using post mortem Xray computer tomography (CT) [5][6]. This technique has two main disadvantages. First, the machining time and raw material have been already spent. Second, X-ray CT is timeconsuming and highly costly and therefore inefficient for mass 
production. Hence, other approaches are found in the literature [5], but rarely and few of those are implemented in real industrial machines [5]. The first method includes highresolution cameras that image each layer completely and image processing algorithms search only surface defects. The second approach involves temperature measurements in the process zone or the melt pool [5][6]. The main disadvantage of this method is that temperature measurements are accessible only at the surface, while estimations of its propagation in depth are based on multiple assumptions, encoded into a model [1][2][8]. The deviations between the real temperatures and predicted ones lead to inaccuracies in quality monitoring. Thus, an in situ and real-time quality monitoring unit remains an open topic and still is of great demand by the AM technology [5].

The present work proposes a novel solution that can be used for in situ and real-time quality monitoring. It is based on detecting the acoustic emission (AE) signals emitted during the powder-bed fusion additive manufacturing (PBFAM) process - a sub-branch of AM technology [5][6]. The AE signal analysis is carried out using machine learning techniques, in particular, spectral convolutional neural network (SCNN). The innovation of applying an active and/or passive AE sensor [9] and ML [10] to monitor the AM process has been internationally recognized by a patent application [11]. Unfortunately, at present, no clear correlation between the $\mathrm{AE}$ signals and the $\mathrm{AM}$ quality has been discovered. However, recent successes of combining AE with state-of-theart signal processing for a highly complex and dynamic process such as friction and wear [12][13], as well as detection of induced micro-cracks [14][15] and laser welding [16][17] motivated this feasibility study to investigate its applicability towards AM. To do so, we combine two extreme technologies: deep neural networks and optical fiber sensor. The former is a state-of-the-art technique in the design of correlation models [18][19] and was applied in a number of practical applications with a high efficiency [20]. The latter, in our work, is a fiber Bragg grating (FBG), which is one of the most sensitive sensor for acoustic and pressure wave's detection [21].

This paper consists of five sections. Section II presents the experimental setup, the materials used, and the data acquisition. Section III describes the signal processing, including $M$-band wavelet spectrogram and SCNN classifier. Section IV reports the classification results and discusses them in terms of unsupervised training of CNNs. It also explores the possibilities of using it in a self-learning process using $\mathrm{AE}$ for AM quality control. Finally, Section V summarizes the findings of our work as well as proposes further perspective of this work.

\section{EXPERIMENTAL SETUP, MATERIAL, AND ACOUSTIC DATASETS}

In this contribution, only a summary of the experimental setup, material, and acoustic datasets are provided, while a complete description is given in [22][23].

Powder-bed fusion additive manufacturing (PBFAM) of CL20ES stainless steel (1.4404 / 316L) powder (particle size:
$10-45 \mu \mathrm{m})$ was performed on a commercially available industrial machine Concept M2 (Concept Laser $\mathrm{GmbH}$, Germany). It was equipped with a fiber laser operated in continuous mode at a wavelength of $1071 \mathrm{~nm}$ with a beam quality $M^{2}=1.02$ and a spot size $d=90 \mu \mathrm{m}$. During the entire process, the parameters of the laser power $P$, hatch distance $h$ and powder layer thickness $l_{t}$ were kept constant at $125 \mathrm{~W}$, $0.105 \mathrm{~mm}$ and $0.03 \mathrm{~mm}$, respectively. The acoustic data collection was performed while manufacturing a workpiece having a cuboid shape with dimensions $10 \times 10 \times 20 \mathrm{~mm}^{3}$, which is shown in Fig. 1, a. The experiment was performed in an $\mathrm{N}_{2}$ atmosphere with an $\mathrm{O}_{2}$ content controlled to be below $1 \%$ throughout the process. The changes in quality were intentionally provoked by changing the manufacturing process by fixing the speed of the scanning laser at 800,500 and $300 \mathrm{~mm} / \mathrm{s}$. The aforementioned speeds were kept constant throughout the entire manufacturing process independently from the number of layers using a machine function. The corresponding energy densities with respect to the scanning speeds were $50,79,132 \mathrm{~J} / \mathrm{mm}^{3}$ [24]. It is known that these regimes provided a range of different pores concentrations inside the workpiece [22][23]. The porosity concentration was controlled and confirmed during visual inspection of the specimen cross-sections and the corresponding light microscopy images are presented in Fig. 1, b-d. The estimated porosity concentrations were: $0.07 \pm 0.02 \%$ for high quality $\left(500 \mathrm{~mm} / \mathrm{s} ; 79 \mathrm{~J} / \mathrm{mm}^{3}\right), 0.3 \pm 0.18 \%$ for medium quality $\left(300 \mathrm{~mm} / \mathrm{s} ; 132 \mathrm{~J} / \mathrm{mm}^{3}\right)$, and $1.42 \pm 0.85 \%$ for poor quality $\left(800 \mathrm{~mm} / \mathrm{s} ; 50 \mathrm{~J} / \mathrm{mm}^{3}\right)$. The sizes and the shapes of the individual pores depend on the energy input, representing different sintering process scenarios. More information about the mechanisms of pores formations under different laser parameters can be found in the work of Bland et al. [24].

For this feasibility study, the acoustic sensing was carried out using a fiber Bragg gratings (FBG) placed directly inside the machine chamber, $20 \mathrm{~cm}$ away from the process zone. Obviously, the position of the FBG and detecting airborne AE signals are certainly not ideal. The reasons are in losses in interface material-air and also in less dense (as compared to the material processed) air environment. Additionally, the sensor placement on the chamber wall is noised by vibrations of printer moving mechanics. However, the results will bring precious information and can be seen as a lower threshold in terms of potential for an in situ and real-time quality control monitoring. As an example, the AE signal of a full highquality layer $\left(79 \mathrm{~J} / \mathrm{mm}^{3}, 500 \mathrm{~mm} / \mathrm{s}\right)$ is shown in Fig. 2,a. The fiber with the FBG sensor inside was pumped with a narrow band laser irradiation at a wavelength of $1547 \pm 0.01 \mathrm{~nm}$ and light power of $4 \mathrm{~mW}$. The FBG sensor provided a $50 \%$ reflectivity of the pumped laser light. More details about FBGs can be found in [21]. The reflected signal was further digitized using high-speed photodiode, connected to data acquisition unit and the data recording software, where both were from Vallen (Vallen Gmbh., Germany). All signals were digitized with the sampling rate of $1 \mathrm{MHz}$. 

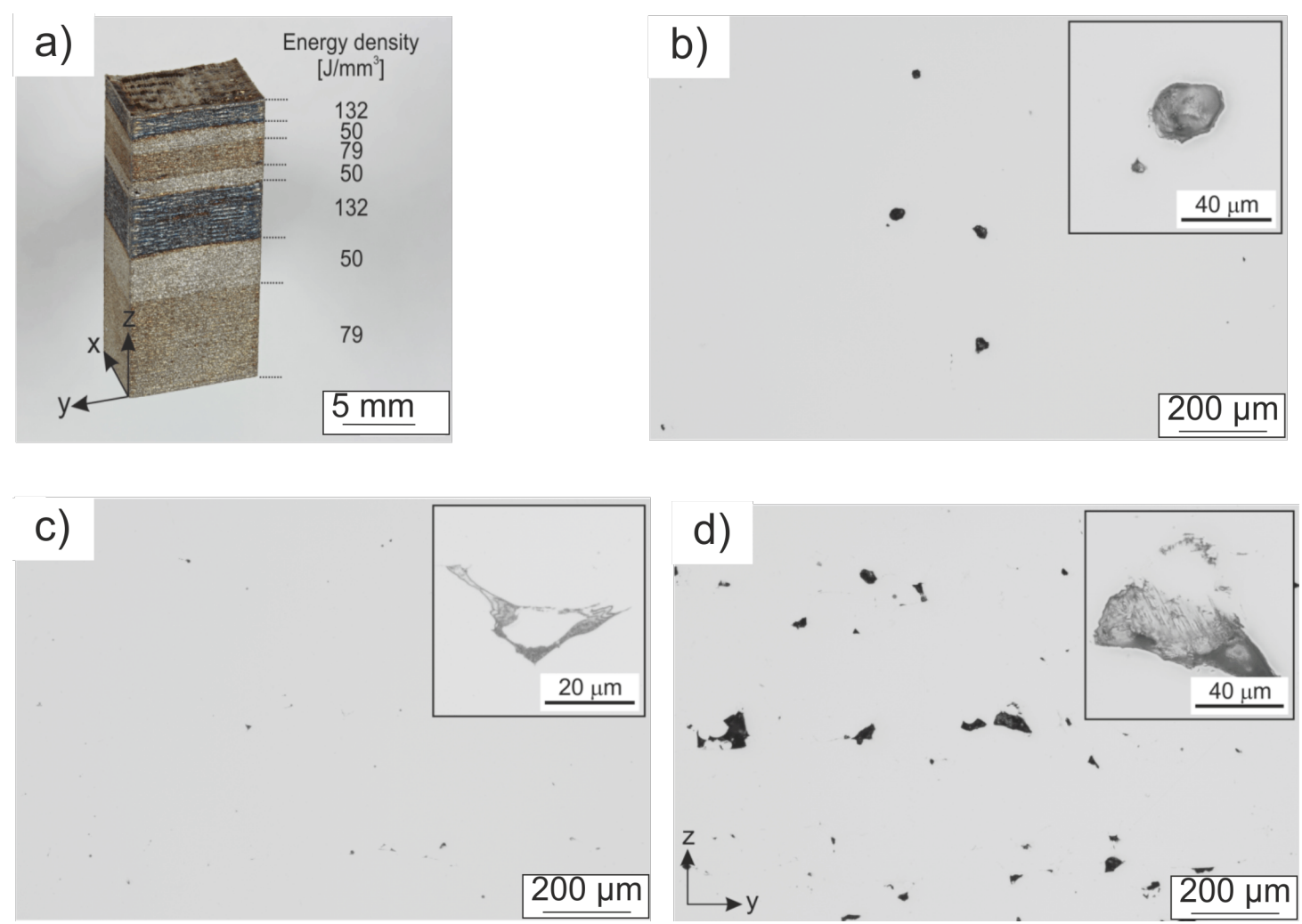

Figure 1 a) Test workpiece produced with three porosity contents; b) - d) Typical light microscope cross-section images of the regions produced with b) $300 \mathrm{~mm} / \mathrm{s}, 132 \mathrm{~mm} 3$ (medium quality), c) $500 \mathrm{~mm} / \mathrm{s}, 79 \mathrm{~mm} 3$ (high quality) and d) $800 \mathrm{~mm} / \mathrm{s}, 50 \mathrm{~mm} 3$ (poor quality). Reprinted by permission from Elsevier License: Elsevier [23].

\section{DATA PROCESSING}

\section{A. Wavelet spectrograms}

We used spectrograms as a feature input for the SCNN classifier providing the search of distinct features in the timefrequency domain. The spectrograms were formed as the relative energies of the narrow frequency bands that were extracted using $M$-band wavelets. $M$-band wavelets are extensions of the traditional wavelet transform [25][26] that, similarly, unfold the signal into a time-frequency space. Its main advantage is in the application of several wavelets at various signal subspaces making them insensitive to shiftinvariance artifacts [26]. The $M$-bands are equivalent to multichannel filtering of the input discrete signal [26] :

$$
\begin{gathered}
\varphi_{j}(n)=\sum_{r} \sqrt{M} h_{0}(r) \varphi(M j n-k), k \subset Z \\
\psi_{j}(n)=\sum_{r} \sqrt{M} h_{m-1}(r) \varphi(M j n-k), k \subset Z
\end{gathered}
$$

where $M$ is the wavelet channels number with a scaling factor $\sqrt{M}, h_{0}, h_{m-2}, h_{m-1}$ are the low, narrow and high pass filters, respectively, $r$ is the filters length, $j$ is the scale and $n$ is a specific time stamp at a given scale. The output of Eqs (1) and (2) are the low, narrow and high frequency contents of the signal at a scale $j$, described by the coefficients $\varphi_{j}(n)$ and $\psi_{j}(n)$. The full signal analysis involves several scales $j$ and the filtering follows the scheme in Fig. 2,b. The extraction of the data on the next scale is carried out using the filtering of the low frequency content, extracted on the previous scale. The final product of multiscale filtering is a pyramidal structure of the narrow frequency bands that capture the local particularities of the signal [25]. More details about wavelet decomposition can be found in [25][26].

In the present work, $M$-band wavelets were obtained using the work from Lin et al. [26], in which, the design of the adaptive wavelets to a given data is carried out using the perfect reconstruction criterion. The four wavelet bands were used here showing an insignificantly low approximation error on the collected AE signals. The corresponding filters coefficients are given in Table I [26].

TABLE I: THE $M$-BAND WAVELETS FILTERS COEFFICIENTS FROM [26] USED FOR THE ANALYSIS.

\begin{tabular}{|c|l|}
\hline \multirow{5}{*}{$h_{0}$} & $-0,019092830 ; 0,0145382757 ; 0,0229906779 ; 0,0140770701 ;$ \\
& 0,$0719795354 ;-0,0827496793 ;-0,1306948909 ;-0,0952930728 ;$ \\
& $-0,1145361261 ; 0,2190308939 ; 0,4145647737 ; 0,4955029828 ;$ \\
& 0,$5616494215 ; 0,3491805097 ; 0,1931394393 ; 0,08571302$ \\
\hline \multirow{5}{*}{$h_{1}$} & $-0,1152813433 ; 0,0877812188 ; 0,1388163056 ; 0,0849964877 ;$ \\
& $-0,443703932 ; 0,1691549718 ; 0,2684936992 ; 0,0722022649 ;$ \\
& $-0,082739818 ;-0,4264277361 ;-0,2550401616 ; 0,6005913823 ;$ \\
& $-0,0115563891 ;-0,1011065044 ; 0,11832820690000 ;-0,1045086525$ \\
\hline \multirow{5}{*}{$h_{2}$} & $-0,0280987676 ; 0,0213958651 ; 0,0338351983 ; 0,0207171125 ;$ \\
& 0,$220295183 ;-0,2088643503 ;-0,3300536827 ;-0,2245618041 ;$ \\
& 0,$5562313118 ;-0,0621881917 ; 0,0010274 ; 0,4477496752 ;$ \\
& $-0,2484277272 ;-0,250343323 ;-0,2048089157 ; 0,2560950163$ \\
\hline \multirow{5}{*}{$h_{3}$} & $-0,0174753464 ; 0,0133066389 ; 0,0210429802 ; 0,0128845052 ;$ \\
& $-0,0918374833 ; 0,0443561794 ; 0,0702950474 ; 0,0290655661 ;$ \\
& $-0,0233349758 ;-0,0923899104 ;-0,0823301969 ; 0,0446493766 ;$ \\
& $-0,1379502447 ; 0,6880085746 ;-0,662289313 ; 0,1839986022$ \\
\hline
\end{tabular}


The relative energies were computed to track the energy redistribution between different frequency bands and were computed as:

$$
\rho_{\text {norm } j, n}=\frac{E_{j}(n)}{E_{\text {total }}}
$$

Where $E_{j}(n)=\psi_{j}(n)^{2}$ is the energy of a specific frequency band at scale $j, n$, as in Eqs (1) and (2), is a time stamp, $E_{\text {total }}$ is a summary of the energies of all frequency bands within the spectrogram. The collection of the products from Eq. (3) from all scales can be unified into a spectrogram by ordering those in time domain. A depictive example is shown in Fig. 2,c, where the spectrogram is reconstructed from the pattern of the signal in Fig. 2,a that is bounded by the red solid marker.

\section{B. Analysis of collected AE signals}

The recorded signals were scanned with two running windows, schematically represented in Fig. 2,a by the green and red solid lines. These windows localize specific patterns of the signal in the time-frequency domain. As seen from Fig. 2,a, both running windows are characterized by longer and shorter time spans and thereafter are referred to as a long (LRW) and a short (SRW) running windows. Both localize acoustic signatures of the momentary laser-material interaction events in the powder-bed. The LRW is a short memory of a number of previous and the current SRWs as shown in Fig. 2,a. The scanning of the entire signal was carried out by a consecutive shift of both running windows and this process is shown in Fig. 2,a, where the solid frames correspond to the previous position of both windows in the time domain, while the dashed ones denote the current position.

As already mentioned, the AM process incorporates multiple events with different time scales and the usage of both windows aimed to provide a multiscale input data for the classifier. Additionally, this approach alleviates the trade between stability and resolution improving both parameters at the same time [27]. On the one hand, the SRW provides a higher resolution of momentary sintering events in the time domain. On the other hand, its short time span makes it sensitive to noises, while LRW is more stable to those. The choice of time spans for both is not obvious and was established via an exhaustive search experiment and the results are discussed below.

In the present investigation, the wavelet spectrograms for SRW and LRW were constructed separately as is discussed in Section III-A. The computational tests in this work include two strategies with different input data for the SCNN classifier. In Strategy 1, both spectrograms (SRW and LRW) were forwarded directly into the SCNN. Each spectrogram included a set of $\rho_{\text {norm } j, n}$ from Eq. (3), ordered in timefrequency domain as is discussed in Section III-A. In Strategy 2, all SRW spectrograms from the training dataset were initially clustered using a basic implementation of the hierarchical agglomerative clustering technique [28]. The clustering was made with respect to Euclidian distance between the relative energies (see $\rho_{\text {norm } j, n}$ from Eq. (3)) of the corresponding frequency bands of SRWs [28]. The idea behind Strategy 2 was to group all SRWs with similar AE characteristics in the same cluster. This digitizes the PBFAM process into a limited number of short-term momentary lasermatter interactions with unique acoustic signatures. A unique label was assigned to each cluster. Further, all LRWs were encoded by the sequence of such labels, substituting the SRWs inside those. In Strategy 2, the inputs for SCNN were both the SRW and the encoded LRW. The architecture of the SCNN was adapted to process the two data flows and is discussed in the next section. The test results for both experiments are presented in Section IV.

It is worthy to note that this work focused on a statistical approach towards the analysis of those patterns. Hence, finding the nature and a physical explanation of the $\mathrm{AE}$ contents of such momentary events were considered as out of the scope of this work.

\section{Spectral convolution neural network}

As already mentioned, SCNN is an extension of the traditional convolutional neural networks (CNNs). The key element of CNN architecture is the convolution layers, in which the input data domain is convolved with a number of local filters, realized as neuronal weights [29]. The output of the convolution layers is a set of perception maps that incorporates the response of each such individual filter to the given data. The construction of the individual filters is a task of statistical learning using a training dataset. The increasing amount of the data, while propagating through the convolution layers, may be reduced. This can be carried out by introducing the merging of the neighbored values in perception maps that are called pooling. The sequence of the convolution and pooling layers serve as a self-feature extraction mechanism and the construction of the optimal features is carried out by tuning the local filters in the convolution layers during the training procedure. Further details about the $\mathrm{CNN}$ architecture can be found in the original work of Krizhevsky et al. [29]. The limits of traditional CNNs are in regular convolution operations that fail to operate on irregular data grids [30], [31]. This problem is solved in the SCNNs, where the spectral graph theory is involved as an external tool to estimate the data irregularity and the convolution operations are adjusted accordingly [31]. In our SCNN, the input features are represented as an input data signal $S$, the elements of which $S_{i}$ are the values of the energies of the individual narrow frequency bands (Section III-A). The input signal is represented as a graph $G=\{V, W\}$, in which the vertecies are samples of the signal. The adjacency matrix $W$ defines a neighborhood of every vertex from $V$, ordering those in the time domain for the signals [31]. In our case, the neighbourhood for each frequency band is defined in the timefrequency domain. On graphs, the convolutions can be efficiently realized as a multiplication in the frequency domain [32], while the transition to the frequency domain is a singular value decomposition of the Laplacian $L$ of the graph $G$ [31], [32]. In this study, the non-normalized Laplacian of $G$ was used that is defined as $L=D-W$, where $D$ is a diagonal weight matrix with elements: $d_{i j}=\sum_{i} w_{j i}$, and $w_{j i}$ are the 

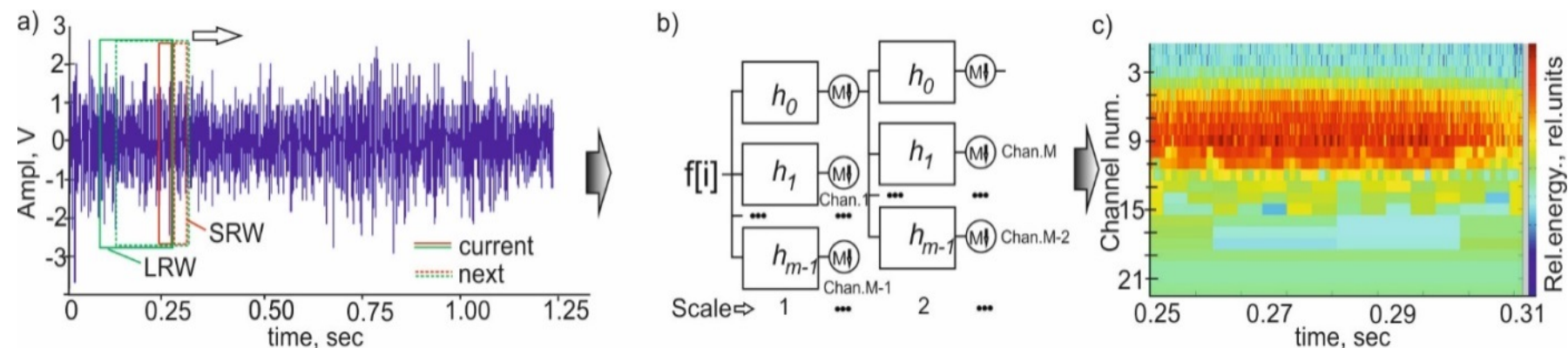

Figure 2 a) A typical AE signal from one complete layer of a high quality $\left(79 \mathrm{~J} / \mathrm{mm}^{3}, 500 \mathrm{~mm} / \mathrm{s}\right)$. SRW and LRW are the short and long running windows that scan the acquired signal; b) the pyramidal extraction of frequency content from the given signal using $M$-band wavelets; c) the complete reconstructed spectrogram from the relative energies of the $M$-band wavelets for the SRW time span bounded in red in Fig. 3a).

elements of $W$. The frequency domain of $S$ is defined by the Fourier transform: $S_{f}=U^{T} S$, where $U$ represents the eigenvectors of the Laplacian $L$ [33]. Its inverse is defined as $S=U S_{f}$ [33]. The perception maps are the product of the convolution and, according to [36], are defined through the spectral domain as: $P_{\text {out }}=U S_{f} k$, where $k$ are the spectral multipliers that link the original and spectral domains [31]. The training back propagation of such a network takes into consideration the gradients of both, the input feature signals and the spectral multipliers: $\nabla S_{j}=U \sum_{i} U^{T} \nabla P_{j i} k_{j i}$, where $j$ specifies the graph signal in the batch and $i$ passes through all the input channels [31]. The back propagation for the spectral multipliers are defined as: $\nabla k_{i}=\sum_{n} U^{T} \nabla P_{n} U^{T} S_{n}$, where $n$ passes through all perception maps. Besides, the spectral multipliers are projected to the neuronal weights as: $\nabla k=F^{T} k$, where $F$ is a smoothing kernel. For pooling of the graph nodes, we used the max pooling strategy since Thomas et al. [27] demonstrated that this strategy is very efficient in acoustic recognition tasks. We used a general realization of SCNN [30] and more mathematical details are in [30]-[33].

The cause of overfitting in the present application is a big dimensionality of the input data (defined by the narrow frequency bands within the given time span of LRWs and SRWs) and the reduced training set (which is suitable for industrial usage). Under these circumstances, the regularization allowed reducing the errors. First, the Laplacian eigenmap regularization of the loss, $£$, for each layer was used [34]: $€=E_{0}+\lambda f(X)^{T} L f($.$) , where L$ is a non-normalized Laplacian, $f($.$) is the layer output [34], \lambda$ is the regularization term that was taken as $10^{-5}$. In addition, the dropout [35] with the filter excluding rate of 0.75 was applied to the last convolution layer. In addition, to process the double inputs (SRW and LRW as discussed in Section III-B), we developed a special configuration of the SCNN.

\section{SCNN classifier for AE analysis}

The developed SCNN model processed two flows of input data and the corresponding scheme is shown in Fig. 3. The classifier included two channels that provided the processing of the information from the LRWs and SRWs. As discussed in Section III-A, in Strategy 1, the LRWs and SRWs were directly fed to the classifier, whereas in Strategy 2, the LRWs were encoded with the labels of the clustered SRWs (See Section III-B). The propagation of the data was separated by the SCNN structure and after one layer was merged in a common convolution layer as shown in Fig. 3. This structure provides the construction of the optimal features during the training procedure. The final classification is carried out by two fully connected layers as schematically shown in Fig. 3. The final results were observed in the output softmax loss layer that returns the label of the porosity concentration.

The domain of the input LRW and SRW spectrograms is a time-frequency domain of the real $\mathrm{AE}$ signal. In this case, the objective of the self-feature extraction was to detect any invariant combinations of individual frequency bands $\left(W_{i}\right.$ in Fig. 3) and their modulations in time ( $W_{j}$ in Fig. 3) that uniquely characterize the $\mathrm{AE}$ signals from each category. The separate channels in the SCNN from Fig. 3 allow extracting separately such invariants.

The SCNN described and all tests were carried out in Visual Studio 2017 and the code was developed based on the library CSharpConvNet. The tests were run on a single i5 processor and $16 \mathrm{~Gb}$ of RAM. The running time for a single classification task was 0.7 second using an un-optimized code.

\section{RESULTS AND DISCUSSIONS}

\section{A. The operating dynamic range}

The level of the background noise produced by the industrial M2 machine was estimated before the data processing using wavelet spectrograms. A representative example of the $\mathrm{AE}$ spectrograms without and with the AM process is shown in Fig. 4,a and 4,b, respectively. The channels in spectrograms from Fig. 4 corresponds to the filters outputs, seen in wavelet decomposition scheme in Fig. 2,b and also denoted in in Fig.2,c. The pseudo frequencies were estimated with respect to the central frequencies of the wavelets [37] that were employed and presented in Table I. As seen from Fig.4, a, the noise from Concept M2 machine was observed in a wide spectral range. Although, the differences brought by the AM process are still visually recognizable when comparing Fig. 4 ,a and $4, \mathrm{~b}$. The added content is mainly observed in the channels $9-15$, which are, therefore, the most suitable ranges for a search of the distinct features. The other ranges of the time-frequency space were selected by taking into consideration the following information. The noises of the 


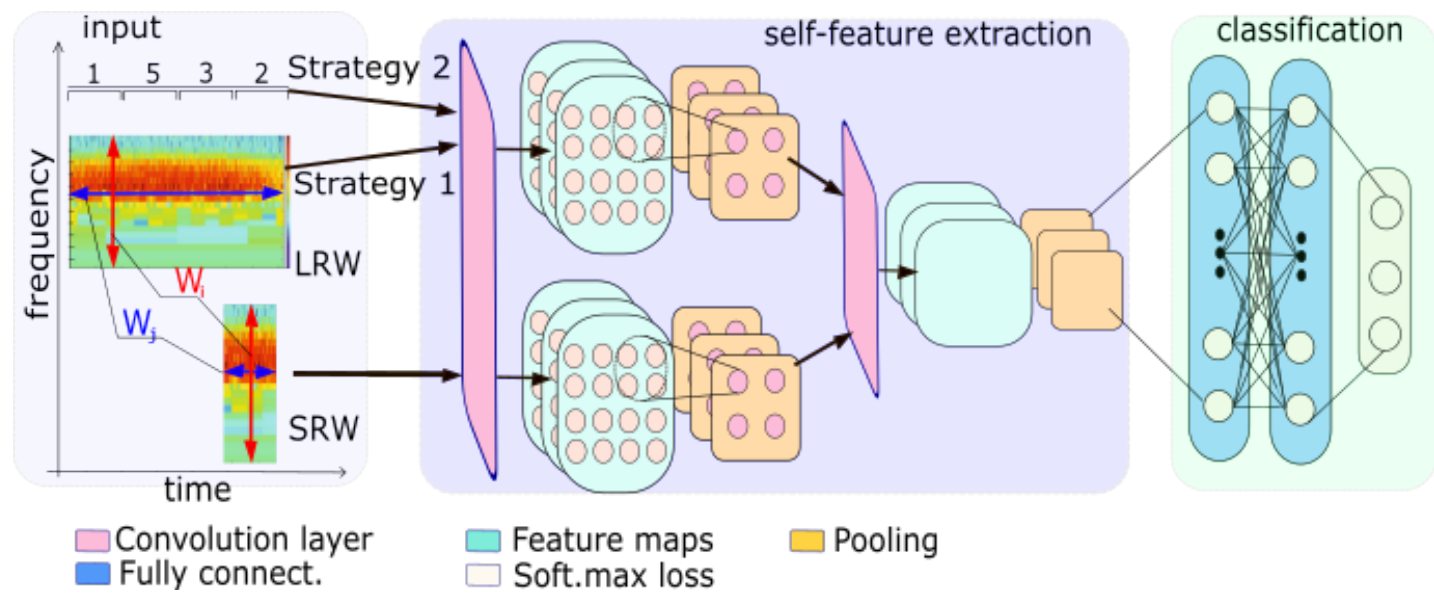

Figure 3: The structure of the SCNN, where $W_{i}$ and $W_{j}$ denote the search direction for the frequency and time shift invariants. Strategy 1 and Strategy 2 denote the input data for the computational tests described in Section III.A.

Concept M2 machine were mainly generated by the moving mechanical parts in conjunction with the turbulences of the gas flow inside the operating chamber. The characteristics of such $\mathrm{AE}$ are well known and are mainly characterized by low frequencies [38]. Taking this information into account, the content from channels 21-25 (Fig. 4,a and 4,b) were excluded from further analysis. Despite the possible noises in higher decomposition channels (greater than channel 9 in Fig.4,a and $4, b)$, those were included into analysis relying on the SCNN to suppress stationary noises [27], [29].

\section{B. Estimation of the spatial resolution of the method}

The maximum resolution of the method and the SCNN classifier was estimated for the two strategies, as is explained in Section III-B. During this the classifier was fed with: 1) both, SRWs and LRWs and 2) SRWs and encoded LRWs. Using the wavelets spectrograms as the input for the SCNN classifier, the time spans for both strategies were estimated through an exhaustive search, while gradually decreasing both time spans and observing the classification accuracy. For Strategy 1, the minimum time spans for the SRWs and LRWs without losses in classification efficiency were reached at 80 and $160 \mathrm{~ms}$, respectively. For Strategy 2 with the encoded LRWs, these values were greater and corresponded to $90 \mathrm{~ms}$ for the SRWs and $1120 \mathrm{~ms}$ for the LRWs. For both strategies, any further decrease of the time spans negatively affected the classification accuracy, while longer time spans did not bring any visible improvements.

The corresponding spatial resolutions of our method are defined by RW time spans. Taking into consideration the known scanning speed $(V)$, laser spot size $(d)$ and a powder layer thickness $\left(l_{t}\right)$ of 30 microns (see Section II), the spatial resolution can be calculated in terms of processing area $\left[\mathrm{mm}^{2}\right]$ and powder volume processed $\left[\mathrm{mm}^{3}\right]$ according to: $A=d \cdot t_{R W} \cdot V\left[\mathrm{~mm}^{2}\right]$, where $t_{R W}$ is either the LRW or SRW time span. The powder volume processed is defined by $A \cdot l_{t}$. The powder volume here, of course, does not characterize the heat affected zone (which may be greater in depth), although was used as a rough estimation of the method precision. The corresponding resolution values are given in Table II. As seen from that data, the spatial resolutions for Strategy 1 varied in the range $4.3-11.6 \mathrm{~mm}^{2}$ and $2.2-5.8 \mathrm{~mm}^{2}$ for the LRW and SRW, respectively. For Strategy 2, these values raised to $30.3-80.6 \mathrm{~mm}^{2}$ and $2.4-6.5 \mathrm{~mm}^{2}$ for the LRW and SRW, respectively. The corresponding values for the powder volume processed are simply the spatial resolution multiplied by the powder layer thickness $\left(l_{t}=30 \mu \mathrm{m}\right)$. We also have to mention that for Strategy 2, the result of spectral graph clustering discovered more than one hundred separate clusters encoding the LRW.

\section{Classification results}

The collected AE signals were divided into two equal datasets without common signals: one for the training and one for testing the classification. The split into train and test sets were done with random separation of the signals. In total, both datasets included 4800 SRWs and 1200 LRWs, extracted from all collected AE signals that corresponded to the three different quality categories. Each category in each dataset was represented by an equal number of samples with 1200 SRWs and 400 LRWs.

The classification test results are presented in Table III. The accuracies for Strategy 1 and Strategy 2 are given in the first line and in bold and italic, respectively. For comparison, we benchmarked the results of our proposed methods with other state-of-the-art machine learning methods, in particular, conventional CNN, art networks Xception [39] and ResNet [40]. These corresponding results are given in bracket in the second line in Table III.

In this table, the accuracy for each quality category is given in rows and can be compared to the ground truth, given in columns. The match of classification with ground truth is in the diagonal cells, while the error structure can be seen from non-diagonal ones. The accuracy was defined as the number of true positives divided by the total number of the tests for the individual categories. As an example for Strategy 1, the AE test data from high quality was classified with an accuracy rate of $78 \%$ and so it has the highest error rate for Strategy 1. The classification errors are shared between the medium and poor quality with error rates of 10 and $12 \%$, respectively. 


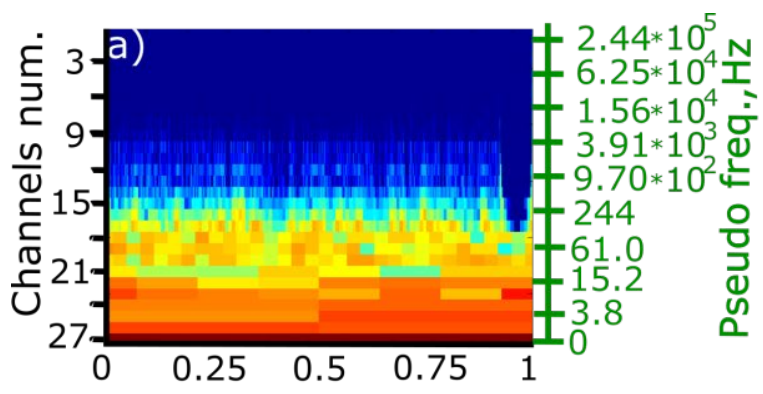

Time, sec.

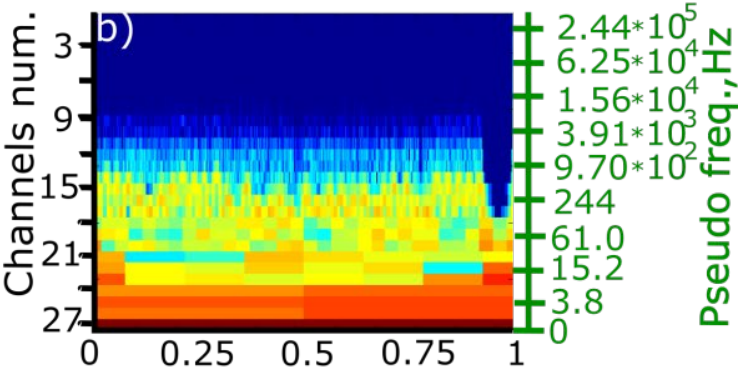

Time,sec.

Figure 4: (a) Spectrograms of Concept M2 machine intrinsic noise background and (b) fragment of the AE signal of the additive process. The pseudo frequencies are computed with respect to the central waves of wavelet filters from Table I according to [37]

TABLE II: THE RESOLUTION OF THE METHOD IN QUALITY ESTIMATION IN TERMS OF PROCESSED SURFACE/VOLUME *

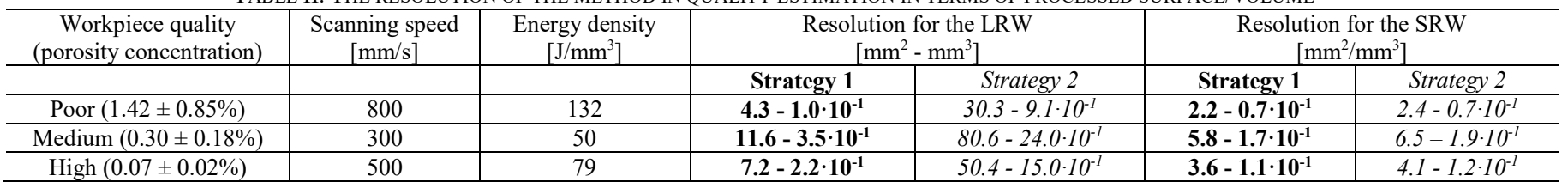

*Strategy 1: SRWs and LRWs spectrograms and Strategy 2: SRWs and encoded LRWs spectrograms. For details, see Section III, B.

As seen from Table III, for Strategy 1, the accuracy ranged from 78 to $91 \%$. As mentioned, the lowest accuracy is for the high quality $(78 \%)$, followed by the poor quality $(87 \%)$ and the medium quality (91\%). It is interesting to note that the highest classification accuracy was achieved for medium quality characterized by the lowest scanning speed (300 $\mathrm{mm} / \mathrm{s})$ and highest energy density $\left(132 \mathrm{~J} / \mathrm{mm}^{3}\right)$. This regime may generate a higher local heating/melting of the material resulting in a higher intensity of the $\mathrm{AE}$ signals and so brings more distinct features in the recorded signals. In contrast, the lowest accuracy was for the high quality produced with the intermediate scanning speed $(500 \mathrm{~mm} / \mathrm{s})$ and energy density $\left(79 \mathrm{~J} / \mathrm{mm}^{3}\right)$. One possible reason may be that the features are a mix of different events from the other two categories. The analysis of the test results indicates that the classification errors are approximately shared equally between the other two categories, thus supporting this hypothesis. One additional reason for errors may be in the strong background noise still present. As seen in Fig. 4,b, the AE contents during the process in the channels $9-15$ are affected by regular fluctuations. This may be due to a machine noise source that was still present in the selected channels of the spectrograms.

For Strategy 2, the classification accuracy ranges between 73 and $86 \%$ as evident from Table III. As for Strategy 1, the lowest classification accuracy is for the high quality (73\%), followed by poor quality (83\%) and medium quality (86\%). The explanations given for Strategy 1 are also valid for Strategy 2. Obviously, the classification accuracy is lower than Strategy 1 despite having longer time spans. These results may be explained by a coarsening of the information in the original spectrogram of LRWs when encoding it with the cluster labels. This may be also a reason that requires longer time spans (see Section IV-B).

The results of the conventional $\mathrm{CNN}$, state-of-the-art networks Xception [39] and ResNet [40] are also in Table III as benchmarks. Both, LRW and the raw AE signals were fed
TABLE III: CLASSIFICATION RESULTS FOR DIFFERENT QUALITY (IN ROWS) VERSUS GROUND TRUTH (IN COLUMNS) FOR STRATEGIES 1 AND $2 *$

\begin{tabular}{|c|c|c|c|}
\hline $\begin{array}{c}\text { Ground truth } \\
\text { Test categories }\end{array}$ & Poor & Medium & High \\
\hline $\begin{array}{c}\text { Poor quality } 1.42 \pm 0.85 \\
\% ; 800 \mathrm{~mm} / \mathrm{s}\end{array}$ & $\begin{array}{c}\mathbf{8 7}-83 \\
(62-66-70)\end{array}$ & $\begin{array}{c}\mathbf{6}-12 \\
(19-15-15)\end{array}$ & $\begin{array}{c}\mathbf{7}-5 \\
(19-19-15)\end{array}$ \\
\hline $\begin{array}{c}\text { Medium quality } \\
0.3 \pm 0.18 \% ; 300 \mathrm{~mm} /\end{array}$ & $\begin{array}{c}\mathbf{5}-6 \\
(25-18-12)\end{array}$ & $\begin{array}{c}\mathbf{9 1}-86 \\
(53-68-75)\end{array}$ & $\begin{array}{c}\mathbf{4}-8 \\
(22-14-13)\end{array}$ \\
\hline High quality & $\mathbf{1 2 - 1 1}$ & $\mathbf{1 0 - 1 6}$ & $\mathbf{7 8 - 7 3}$ \\
$0.07 \pm 0.02 \% ; 500 \mathrm{~mm} / \mathrm{s}$ & $(20-21-17)$ & $(17-25-23)$ & $(63-54-60)$ \\
\hline
\end{tabular}

The color fill encodes the match of the test result to the ground truth. The results in each cell are given in format: Strategy 1 - Strategy 2

(LRW as input using conventional CNN - LRW as input using Xception CNN - LRW input using ResNet)

to those. The structure of training and test sets was identical to the SCNN. It is evident from Table III that the classification accuracies achieved by the three methods are significantly worse ( $>11 \%$ differences) than the method proposed. The CNNs are also famous for processing the raw data, as construct the best-matched features on their own [29],[30]. This minimizes the algorithm development or preparation time, excludes the inefficient organization of the input data and thus is important for industrial applications. For this reason, the same neural networks were tested with the raw $\mathrm{AE}$ signals as input. The results obtained were worse than the ones from Table III and are not shown here. The reasons may be in high noise levels in AE signals, that require the expansion of the training set. At present, the performance of the networks with the raw data is worse than using our model, although the models' optimizations for direct raw data input are planned as future work.

The results for conventional CNN were taken from [23], which was used without GPU embedding. The recent state of the art networks Xception [39] and ResNet [40] were constructed using Pythorch library 0.4.1. Each network included four convolutions. Both were used in a CPU and GPU versions. The GPU embedding used NVidia GPU 1070 Max-Q, while the PC included i7 processor with 12 cores. It 
can be seen from the comparison in Table III that all these architectures showed lower classification results as compared to our SCNN approach. At the same time, a higher processing speed for Pythorch realizations was observed. The GPU embedded CNNs was $50 \mathrm{~s}$ for the entire training and $10 \mathrm{~ms}$ for single classification operation. In the case of the CPU versions, these times increased by factor five.

Our SCNN was used with a CPU. Hence, the training and computational time for each classification operation for our realization were distinctly higher, taking $1.5 \mathrm{~h}$ for the entire training and $70 \mathrm{~ms}$ for single classification operation. However, it is important to mention that, for industrial applications where high dynamics and complex processes are involved, this $1.5 \mathrm{~h}$ training time is not prohibitive. Even the classification time is not an obstacle since today; there is no monitoring system that allows stopping the process based on quality control directly on the workpiece.

Based on Table III, we demonstrated that combining highly sensitive AE sensors and using an SCNN to classify workpiece qualities in terms of porosity is feasible. Moreover, the results presented can be enhanced by increasing the sensor's sensitivity, their positioning, as well as optimizing the signal processing. Additionally, this work demonstrates that the continuous PBFAM process can be divided into a finite number of momentary events that possess unique acoustic signatures. Those signatures can be read by the machine learning framework and interpreted in terms of quality.

The technical aspects are in the computational speed. Processing of the collected dataset with conventional CNNs using Pythorch library and GPU allowed reaching real-time for quality monitoring. The market availability of the GPU hardware allows the fast transfer of these methods into mass production lines. Based on the test results using GPU the porting of our SCNN to GPU is also planned in the future.

Additionally, the approach proposed has a large potential to be a part of the fully automated quality closed loop control systems. In this case, the more detailed quality grading could be a feedback to the control, indicating the direction of the process parameters tuning.

\section{CONCLUSIONS AND FUTURE WORK}

This work presents the results of a feasibility study for in situ and real-time quality monitoring in additive manufacturing (AM). To achieve this goal, a Concept M2 industrial machine was used to produce a workpiece with three quality categories (high, medium and poor qualities). The qualities were defined with respect to the porosity concentration and were obtained with specific process parameters. The laser power was set to $P=125 \mathrm{~W}$, the hatching distance to $h=0.105 \mathrm{~mm}$ and the layer thickness to $t=0.03 \mathrm{~mm}$. The qualities were obtained using different scanning velocities according to: high quality $(0.07 \pm 0.02 \%$ porosity; $\left.500 \mathrm{~mm} / \mathrm{s} ; \quad 79 \mathrm{~J} / \mathrm{mm}^{3}\right)$; medium quality $\left(0.3 \pm 0.18 \% ; 300 \mathrm{~mm} / \mathrm{s} ; 132 \mathrm{~J} / \mathrm{mm}^{3}\right)$; and poor quality $\left(1.42 \pm 0.85 \% ; 800 \mathrm{~mm} / \mathrm{s} ; 50 \mathrm{~J} / \mathrm{mm}^{3}\right)$.

Our innovative approach combines a fiber Bragg grating (FBG) as an acoustic emission sensor with machine learning technique to build a classifier to differentiate those signals. Wavelet decomposition was used to digitize the data in the time-frequency domain and to extract features. Two computational strategies were carried out. In Strategy 1, wavelet sonograms were directly fed into the classifier, while in Strategy 2, it was substituted by the code of the sequence of the momentary sintering events.

The results of the classification tests showed that the three laser regimes, resulting in different pores concentrations, can be recognized with confidences as high as 78 to $91 \%$ for Strategy 1 . The corresponding spatial resolutions varied from $4.3-11.6 \mathrm{~mm}^{2}$ and $2.2-5.8 \mathrm{~mm}^{2}$ for the LRW and SRW, respectively. For Strategy 2, all SRW spectrograms from the training dataset were initially clustered using a basic implementation of the hierarchical agglomerative clustering technique. The classification accuracies ranged from 73 to $86 \%$ and the spatial resolution was comprised between $30.3-$ $80.6 \mathrm{~mm}^{2}$ and $2.4-6.5 \mathrm{~mm}^{2}$ for the LRW and SRW, respectively. Obviously, this approach showed a lower classification accuracy as compared to Strategy 1. The explanation of this may lay in the tremendous reduction in the data details when encoding the LRWs.

Despite a decrease in the classification accuracy, Strategy 2 showed that the PBFAM process can be divided into a finite number of groups for momentary laser-material interaction events that possess unique acoustic signatures. The combinations of those events during a continuous AM process can be used to differentiate different regimes that potentially provoke low manufacturing quality.

Based on the results presented, we can conclude that the acoustic emission provides distinct features from different manufacturing quality. The features extracted are differentiable within machine learning technique despite the high noise levels of the industrial AM machines.

The biggest achievement of our approach using both strategies is the sub-layer resolution in quality estimates, which is not reached with existing image-based methods [5].

In the near future, several enhancements will be implemented in three main areas which are the industrial machine, the sensors and the signal processing.

For the machine, the next significant progress of our technology aim to be adapted for in situ and real-time usage within existing commercial machines without significant modifications. This is supported by the commercial availability of both, FBGs and GPUs, which will allow a fast embedding into existing manufacturing facilities.

As far as the FBG are concerned, two major improvements will be applied. The first one is related to the positioning of the FBG and the second is an optimization of its spectral characteristics.

Finally, in terms of signal processing, five areas will be improved. They are the followings: a more efficient noise suppression inside the network, an input data dimensionality reduction, some investigations of conditions, determine the limits for over fit and finally use self-learning of new events. The later has been already conducted by using reinforcement learning [41]. 


\section{REFERENCES}

[1] W.E. King, A.T. Anderson, R.M. Ferencz, N.E. Hodge, C. Kamath, S.A. Khairallah, A.M. Rubenchik, Laser powder-bed fusion additive manufacturing of metals; physics, computational, and materials challenges, Appl. Phys. Rev, 2 (2016) 041304, http://dx.doi.org/10.1063/1.4937809.

[2] S. Guessasma, W. Zhang, J. Zhu, S. Belhabib, H. Nouri, Challenges of additive manufacturing technologies from an optimisation perspective, Int. J. Simul. Multisci. Des. Optim. 6 (2015) A9, http://dx.doi.org/10.1051/smdo/2016001

[3] Y.W. Zhai, D.A. Lados, J.L. Lagoy, Additive manufacturing: making imagination the major limitation. JOM, 66(5) (2014) 808-816, http://dx.doi.org/10.1007/s11837-014-0886-2.

[4] S.A. Khairallah, A.T. Anderson, A. Rubenchik, W.E. King, L. Livermore, Laser powder-bed fusion additive manufacturing: Physics of complex melt flow and formation mechanisms of pores, spatter, and denudation zones, Acta Mater. 108 (2016) 36-45, http://dx.doi.org/10.1016/j.actamat.2016.02.014.

[5] S.K. Everton, M. Hirsch, P. Stravroulakis, R.K. Leach, A.T. Clare, Review of in-situ process monitoring and in-situ metrology for metal additive manufacturing, Mater. Design, 95(5) (2016) 431-445, http://dx.doi.org/10.1016/j.matdes.2016.01.099.

[6] G. Tapia, A. Elwany, A review on process monitoring and control in metal-based additive manufacturing, J. Manuf. Sci. Eng 136(6) (2014) 060801 http://dx.doi.org/10.1115/1.4028540

[7] W. Shifeng, L. Shuai, W. Qingsong, C. Yan, Z. Sheng, S.Yusheng, Effect of molten pool boundaries on the mechanical properties of selective laser melting parts. J. Mater. Process. Tech. 214(11) (2014) 2660-2667, http://dx.doi.org/10.1016/j.jmatprotec.2014.06.002.

[8] T. Polivnikova, Study and modelling of the melt pool dynamics during selective laser sintering and melting, $\mathrm{PhD}$ Thesis $\mathrm{N}^{\circ}$ 6826, Ecole Polytechnique Federale de Lausanne (EPFL), (2015), http://dx.doi.org/10.5075/epfl-thesis-6826

[9] T. Purtonen, A. Kalliosaari, A. Salminen, Monitoring and adaptive control of laser processes, Phys. Procedia 56 (2014) 1218-1231, http://dx.doi.org/10.1016/j.phpro.2014.08.038.

[10] Farson, Dave F., Kirk T. Kern, and Kenneth S. Fang. "Neural network classification of laser welds from acoustical signals." The Journal of the Acoustical Society of America 87.S1 (1990): S35-S36.APA

[11] K. Wasmer, S.A. Shevchik, F. Vakili-Farahani, S. Vaucher, and G. Violakis, In-situ and real time quality control in additive manufacturing process EP no. 3258219A1, WO no. 2017216059A1, June 2016.

[12] F. Saeidi, S.A. Shevchik and K. Wasmer Automatic Detection of Scuffing Using Acoustic Emission, Tribol. Int., 94, (2016), 112-117, http://dx.doi.org/10.1016/j.triboint.2015.08.021.

[13] S.A. Shevchik, F. Saeidi, B. Meylan and K. Wasmer Prediction of Failure in Lubricated Surfaces Using Acoustic Time-Frequency Features and Random Forest Algorithm, IEEE Trans. Ind. Informat., 13(4), (2017), 1541-1553, http://dx.doi.org/10.1109/TII.2016.2635082.

[14] S.A. Shevchik, B. Meylan, A. Mosaddeghi and K. Wasmer Acoustic Emission for In Situ Monitoring of Solid Materials Pre-weakening by Electric Discharge: A Machine Learning Approach, IEEE Access, 6(1), (2018), 40313-40324, https://doi.org/10.1109/ACCESS.2018.2853666.

[15] S.A. Shevchik, B. Meylan, G. Violakis, and K. Wasmer 3D Reconstruction of Cracks Propagation in Mechanical Workpieces Analyzing Non-Stationary Acoustic Mixtures, Mech. Syst. Signal Process. 119, (2019), 55-64, https://doi.org/10.1016/j.ymssp.2018.09.022.

[16] K. Wasmer, T. Le-Quang, B. Meylan, F. Vakili-Farahani, M:P: Olbinado, A. Rack, and S.A. Shevchik, Laser Processing Quality Monitoring by Combining Acoustic Emission and Machine Learning: A High-Speed X-Ray Imaging Approach, Procedia CIRP, 74, (2018), 654658, https://doi.org/10.1016/j.procir.2018.08.054.

[17] T. Le-Quang, S.A. Shevchik, B. Meylan, F. Vakili-Farahani, M.P. Olbinado, A. Rack, and K. Wasmer, Why Is In Situ Quality Control of Laser Keyhole Welding a Real Challenge? Procedia CIRP, 74, (2018), 649-653, https://doi.org/10.1016/j.procir.2018.08.055.

[18] J. Schmidhuber, Deep learning in neural networks: An overview, Neural Networks, J. Neu. Net. 61 (2015) 85-117, http://dx.doi.org/10.1016/j.neunet.2014.09.003.

[19] X. Chang, Y. Yu, Y. Yang, E. P. Xing, Semantic Pooling for Complex Event Analysis in Untrimmed Videos, IEEE Transactions on Pattern
Analysis and Machine Intelligence, vol. 39(8) (2017) 1617-1632, https://dx.doi.org/10.1109/TPAMI.2016.2608901

[20] O.A. Hamid, A. Mohamed, H. Jiang, G. Penn, D. Yu, Convolutional neural networks for speech recognition, EEE/ACM Trans. Audio, Speech, Language Process. 22(10) (2014) 1533-1545, http://dx.doi.org/10.1109/taspl.2014.2339736.

[21] R. Kashyap "Fiber Bragg Gratting", 2nd Ed. Elsevier, ISBN: 978-0-12372579-0. (2010)

[22] K. Wasmer, C. Kenel, C. Leinenbach and S.A Shevchik, In situ and realtime monitoring of powder-bed AM by combining acoustic emission and artificial intelligence, M. Meboldt and C. Klahn (eds.), AMPA2017, Springer International Publishing AG 2018, http://dx.doi.org/10.1007/978-3-319-66866-6_20

[23] S.A Shevchik, C. Kenel, C. Leinenbach and K. Wasmer, Acoustic emission quality monitoring in additive manufacturing using spectral convolutional neural networks, Add. Manf. 21, 2018, 508-604, https://doi.org/10.1016/j.addma.2017.11.012.

[24] S. Bland, N.T. Aboulkhair, Reducing porosity in additive manufacturing, Met. Powder Rep. 70(2) (2014) 77-86, http://dx.doi.org/10.1016/j.mprp.2015.01.002.

[25] Daubechies I., Ten Lectures on Wavelets; CBMS-NSF Regional Conference Series in Applied Mathematics (1992), http://dx.doi.org/10.1137/1.9781611970104

[26] T. Lin, S. Xu, Q. Shi, and P. Hao. An algebraic construction of orthonormal M-band wavelets with perfect reconstruction, Appl. Math. Comput. 172(2) 717-730, http://dx.doi.org/10.1016/j.amc.2004.11.025

[27] S. Thomas, S. Ganapathy, G. Saon, H. Soltau, Analyzing convolutional neural networks for speech activity detection in mismatched acoustic conditions, Acoustics, Speech and Signal Processing (ICASSP), 2014 IEEE International Conference on Acoustics, Speech and Signal Processing (ICASSP), http://dx.doi.org/10.1109/ICASSP.2014.6854054

[28] L. Rokach, M. Oded, Clustering methods, Data mining and knowledge discovery handbook. Springer US, pp. 321-352 (2005)

[29] A. Krizhevsky, I. Sutskever, G.E. Hinton, ImageNet classification with deep convolutional neural networks, Advances in Neural Information Processing Systems 25 (NIPS 2012), Lake Tahoe, Nevada, (2012).

[30] M. Henaff, J. Bruna, Y. LeCun, Deep convolutional networks on graph structured data. CoRR, abs/1506.05163, (2015)

[31] Jean Gallier, Spectral Theory of Unsigned and Signed Graphs Applications to Graph Clustering: a Survey, arXiv:1601.04692.

[32] I. Safro, Comparison of coarsening schemes for multilevel graph partitioning. In Int. Conf. Learning and Intelligent Optimization, pages 191-205. Springer-Verlag, 2009.

[33] R. Bracewell, The Fourier Transform \& Its Applications, McGraw, 1999.

[34] J. Weston, F. Ratle, H. Mobahi, R. Collobert, Deep learning via semisupervised embedding. In Neural Networks: Tricks of the Trade, pp. 639-655. Springer, 2012

[35] N. Srivastava, G. E. Hinton, A. Krizhevsky, I. Sutskever, R. Salakhutdinov, Dropout: a simple way to prevent neural networks from overfitting. Journal of Machine Learning Research (JMLR), 15(1):19291958,2014

[36] I. Safro, Comparison of coarsening schemes for multilevel graph partitioning. In Int. Conf. Learning and Intelligent Optimization, pages 191-205. Springer-Verlag, 2009.

[37] P. Abry, Ondelettes et turbulence: Multirésolutions, algorithmes de décomposition, invariance d'échelles, Diderot Editeur, Paris (1997), ISBN: 2-84134-064-3.

[38] Handbook of Noise and Vibration Control, M.J. Crocker (Eds.), John Wiley \& Sons, New Jersey, 2007, ISBN: 978-0-471-39599-7, http://dx.doi.org/10.1121/1.2973236.

[39] F. Chollet, Xception: Deep Learning with Depthwise Separable Convolutions (2018),arXiv:1610.02357.

[40] K. He, X. Zhang, S. Ren, and J. Sun. Deep residual learning for image recognition. arXiv preprint (2015), arXiv:1512.03385.

[41] K. Wasmer, T. Le-Quang, B. Meylan and S.A. Shevchik, In Situ Quality Monitoring in AM Using Acoustic Emission: A Reinforcement Learning Approach, J. Mat. Eng. Perf., 28, (2019), 666-672, https://doi.org/10.1007/s11665-018-3690-2. 


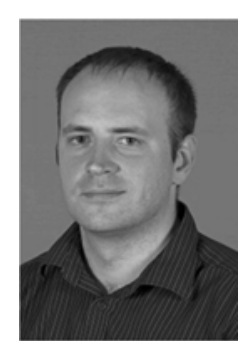

Shevchik Sergey received the engineering degree in control from Moscow Engineering Physics Institute in 2003 and $\mathrm{PhD}$ degree in biophotonics from General Physics Institute, Russia, in 2005 where he stayed as a post-doctoral researcher until 2009. Between 2009 and 2012, he was with Kurchatov Institute, Russia developing image processing for human machine interfaces. In $2012-2014$, he worked at University of Bern focusing on computer vision systems and multi view geometry. Since 2014, he is a scientist at the Swiss Federal Laboratories for Material Science and Technology (Empa), developing signal processing and machine learning for industrial automatization. His research interests are in image/signal processing, pattern recognition and machine learning.

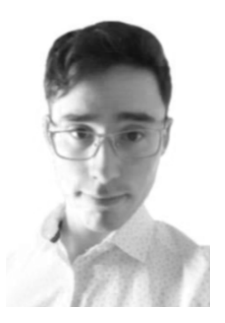

Giulio Masinelli received his B.Sc. degree in Electrical Engineering from University of Bologna, Italy in 2017. In 2019, he is a Master student in Electrical Engineering (with data science specialization) at EPFL, Lausanne, Switzerland. He is working as intern at Swiss Federal Laboratories for Material Science and Technology (Empa), mainly developing machine-learning algorithm for data analysis and industrial automation. His research interests are in signal processing and machine learning with emphasis on deep learning.

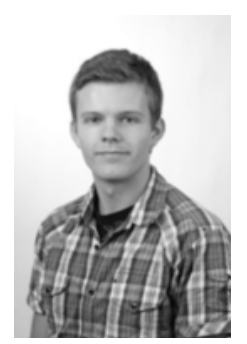

Christoph Kenel received the B.Sc, M.Sc and Ph.D. degrees in materials science and engineering from ETHZ, Zürich, Switzerland, in 2011, 2013 and 2016, respectively. $\mathrm{He}$ is currently with Northwestern University, Chicago, USA, as a Postdoctoral Researcher. His research interests include additive manufacturing of metals and composites using beambased and ink-extrusion-based processing techniques and in situ synchrotron X-ray process studies.

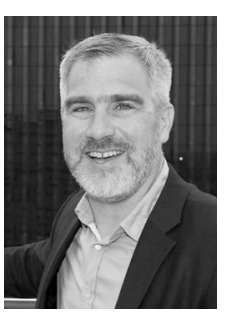

Christian Leinenbach received his M.Sc. in Materials Science and Engineering from the University of Saarbrücken (DE) in 2000 and his $\mathrm{PhD}$ from the University of Kaiserslautern (DE) in 2004. He has been working at Empa since 2005, currently in the position as Head of the Alloy Design for Advanced Processing Technologies (ADAPT) Group in the Laboratory for Advanced Materials Processing in Dubendorf and Thun, Switzerland. In addition, he is adjunct lecturer for materials processing at EPFL, Lausanne, Switzerland.

Dr. Leinenbach's research work has been focusing on the simulation-assisted development/optimization and microstructure design of structural alloys and metal-matrix composites, mainly for beam-based additive manufacturing and joining applications. Besides, he is interested in the characterization of the influence of advanced processing technologies on the microstructure and properties of complex structural materials.

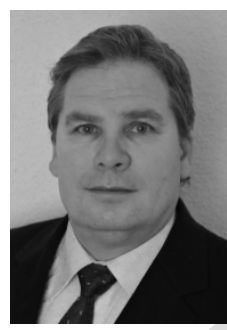

Kilian Wasmer (M'15) received the B.S. degree in mechanical engineering from the Applied University, Sion, Switzerland and Applied University, Paderborn, Germany in 1999. He received his Ph.D. degree in mechanical engineering from Imperial College London, Great Britain, in 2003.

$\mathrm{He}$ joined Empa (Swiss Federal Laboratories for Materials Science and Technology), Thun, Switzerland, in 2004 to work on control of crack propagation in semi-conductors. Currently, he leads the group of dynamical processes in the Laboratory for Advanced Materials Processing (LAMP). His research interests include materials deformation and wear, crack propagation prediction and material-tool interaction in particular laser material processing (e.g. welding, cutting, drilling, marking, cladding, additive manufacturing, etc.). In the last years, he has focused his work on process development, process monitoring and quality control via in situ and real-time observation of complex processes using acoustic and optical sensors in various fields such as in tribology, fracture mechanics and laser processing.

Dr. Wasmer is in the director committee for additive manufacturing of Swiss Engineering. He is also member of Swiss tribology, European Working Group of Acoustic Emission (EWGAE), Swissphotonics and Deutsche Gesellschaft für Zerstörungsfreie Prüfung (DGZfP). 